\title{
BIM-modeling and programming of curved concrete walls for 3D-printed construction
}

\author{
Juan Gabriel Arboleda-Pardo \\ Universidad del Bío Bío | Chile | jg.arbpard@gmail.com \\ Rodrigo García-Alvarado \\ Universidad del Bío Bío | Chile | rgarcia@ubiobio.cl
}

Alejandro Martínez-Rocamora

Universidad de Sevilla | España | rocamora@us.es

\begin{abstract}
This article presents the parametric design and modeling in BIM of curved walls for 3d-printed construction in concrete, seeking to manage the reduction of materials and construction execution times, and enhance its architectural expression. The process described here is structured in the following phases: (i) conceptual preliminary design exploration, defining formal parameters in Revit, (ii) parametric modeling with Dynamo and Revit, (iii) integration of structural validation and printing programming of the robotic arm, and examples of execution with 3D-printed construction.
\end{abstract}

Keywords: BIM; Parametric programming; 3D-printed Construction; Curved wall; Digital fabrication.

\section{INTRODUCCIÓN}

La impresión 3D ha revolucionado el mundo, reduciendo tiempos y procesos de fabricación (Berman, 2012). Esta tecnología ha evolucionado con el tiempo y se ha aplicado en distintas áreas de la industria, entre ellas la edificación, en donde se conoce como construcción impresa 3D (Perkins \& Skitmore, 2015). Dicha tecnología se basa en la deposición tridimensional progresiva de capas de material con control digital (Bos, Wolfs, Ahmed, \& Salet, 2016).

En el ámbito de investigación en arquitectura, la construcción impresa 3D se ha convertido en un foco de interés, ya que se considera que puede revolucionar la industria debido a las ventajas que implica, como reducción de tiempos de construcción e implícitamente los costos relacionados (Buswell, Soar, Gibb, \& Thorpe, 2007). Diversos estudios se centran en el diseño de mezclas de concreto para construcción impresa (Craveiro, Nazarian, Bartolo, Bartolo, \& Pinto Duarte, 2020), estudio del dimensionamiento y geometría de las boquillas, velocidad de impresión, y desarrollo de mezclas cementicias para mejorar la calidad de la extrusión para impresión 3D y determinar su relación de flujo (Nair, Panda, Santhanam, Sant, \& Neithalath, 2020). Otros estudios abordan las fallas estructurales de los elementos impresos durante el proceso de fabricación por extrusión (Muthumanickam et al., 2020; Wolfs \& Suiker, 2019; Zareiyan \& Khoshnevis, 2017).

La implementación de la construcción impresa 3D implica ajustes en la normalización y regulación de algunas condiciones constructivas, sistemas y productos relacionados. Además, para que la tecnología de construcción impresa se establezca como una opción real, es necesario que presente ventajas comerciales y constructivas que la hagan competitiva (García-Alvarado, 2018). Para ello, es necesario seguir procesos estandarizados, abrir los formatos de datos, ajustar los procesos orientados al diseño y planificación de la construcción, entre otras iniciativas (Teizer, Blickle, King, Leitzbach, \& Guenther, 2016). Lo anterior se suma a la necesidad de hacer la construcción cada vez más segura, debido a que es una de las industrias más peligrosas por el riesgo generado a sus trabajadores por las actuales necesidades en edificación (Salminen \& Tallberg, 1996; Sorock, O'Hagen Smith, \& Goldoff, 1993). La evolución hacia la construcción impresa $3 \mathrm{D}$ con equipos robotizados lograría hacer estos procesos más eficientes y seguros (Figura 1).

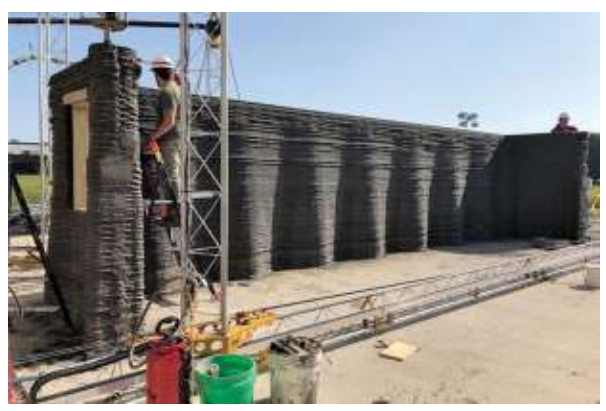

Figura 1: Construcción impresa de barraca militar-EE.UU. (Fuente: www.enr.com/articles/45002-army-researchers-refine3d-printed-concrete-barracks)

Adicionalmente, la construcción impresa 3D progresivamente se está convirtiendo en una alternativa sustentable comparada con la construcción tradicional. Algunos autores señalan que un amplio conocimiento de los beneficios de esta tecnología puede transformar o mejorar la construcción tradicional, potenciando el diseño 
y conduciéndolo a ser más sustentable (Uppala \& Tadikamalla, 2017).

La automatización de la construcción impresa implica ampliar el campo de en arquitectura y construcción y dirigirla hacia la programación, codificación y fabricación digital. El diseño paramétrico y la programación paramétrica pueden facilitar la comunicación directa con robots, lo cual es fundamental para optimizar los procesos (Monedero, 2000). Muchos estudios sobre construcción impresa 3D han contribuido en este aspecto abordando el potencial de esta tecnología, exponiendo y evaluando sus posibilidades de aplicación (Hager, Golonka, \& Putanowicz, 2016; Tay et al., 2017; Yin, Qu, Zhang, \& Lim, 2018), así como los códigos de construcción y normas aplicables y los equipos y tecnología disponibles en el mercado (Mechtcherine et al., 2019).

Los temas actuales de interés para las tecnologías aplicadas al diseño y construcción son el desarrollo de formas complejas y su viabilidad constructiva. La construcción impresa tiene un gran potencial para lograr formas libres y geometrías complejas con altos niveles de precisión, control de obra y reducción de materiales. Sin embargo, comparativamente esta tecnología resulta mucho más costosa que los métodos constructivos convencionales de edificaciones ortogonales (Yin et al., 2018). La impresión de elementos inclinados, al igual que de elementos curvados verticalmente (con inclinación variable en el elemento con respecto a la vertical) implica una deposición de capas con un ligero desplazamiento entre ellas con respecto a la vertical. El ángulo que se genera entre los bordes de capas sucesivas de un mismo lado se denomina ángulo de proyección. En el campo del diseño industrial se han realizado algunas investigaciones de impresión $3 \mathrm{~d}$ de variados materiales sobre superficies y volúmenes arbitrarios con la exploración del diseño paramétrico, aunque propias de otras áreas de investigación, el proceso logrado tiene una alta aplicabilidad en el campo de la arquitectura y construcción (Coulter, Coulter, Papastavrou, \& lanakiev, 2018; Cui \& Yue, 2020; Suzuki, Yatani, Gross, \& Yeh, 2018).

\section{ANTECEDENTES DE INVESTIGACIÓN}

\section{ESTADO DEL PROBLEMA}

La construcción de formas complejas continúa siendo un reto para la arquitectura debido a su potencial estético. Aunque las herramientas digitales actuales han facilitado esta brecha desde el punto de vista del diseño, para el caso de la construcción impresa 3D debe integrarse con la ejecución, particularmente en la construcción de elementos de doble curvatura.

La exploración de geometrías no tradicionales ha sido abordada progresivamente con la impresión de superficies curvas o combinaciones de tramos curvos y rectos. Por ejemplo, el prototipo de vivienda para el Solar Decathlon África, celebrado en Marruecos en 2019, recurrió al diseño paramétrico para evaluar múltiples alternativas formales con geometrías basadas en trazados combinados de curvas y rectas y encuentros curvos. Este diseño de vivienda adaptativo cambiaba su configuración en función de los parámetros de superficie, emplazamiento, relaciones visuales y espaciales al interior de la vivienda. Al final se eligió una alternativa optimizada en cuanto al límite máximo de utilización de concreto y al menor recorrido de la impresora (Sánchez Gómez, Alapont Ramón, Bono Cremades, \& Catalán Tamarit, 2020). Una muestra de la construcción impresa del prototipo se ve en la fig.2.

La necesidad de explorar soluciones formales más complejas ha llevado a que algunas investigaciones sobre la tecnología para impresión $3 \mathrm{D}$ con concreto hayan abordado el desarrollo de formas curvas y geometrías complejas como cúpulas y columnas helicoidales (Cho, Kruger, Zeranka, \& Zijl, 2019). Se han estudiado formas curvas, onduladas, con algún grado de complejidad, con patrones interiores para generar cavidades que aligeran el peso (Izard et al., 2017), e inclusive la construcción impresa de un panel sándwich prefabricado de doble curvatura generado a partir de capas curvadas con aplicabilidad en construcciones de gran envergadura (Lim et al., 2016).

Lograr geometrías complejas con construcción impresa es un reto relevante debido al proceso que implica con el depósito de capas paralelas, además con la impresión desfasada con la vertical para lograr superficies inclinadas. Algunos investigadores estudiaron superficies de doble curvatura, es decir, curvas en sentido vertical y horizontal, que implican un ángulo de proyección variable entre capas, usándolas en componentes de muros prefabricados no convencionales mediante diseño paramétrico integrado, lo que les permitió seleccionar algunas de las piezas para construcción impresa con varias alternativas en su configuración (O. Kontovourkis \& Tryfonos, 2018). Otros autores centraron su trabajo en muros y elementos modulares estructurales de formas complejas elaborados con concreto de alto desempeño (Gosselin et al., 2016).

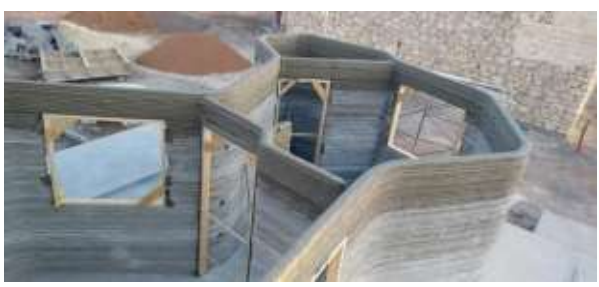

Figura 2: Prototipo impreso para el Solar Decathlon África 2019 (Fuente: (Sánchez Gómez et al., 2020)).

Sin embargo, se ha observado poco trabajo con respecto a la integración coherente y robusta del diseño paramétrico en el diseño digital para lograr procesos de construcción automatizados (O. Kontovourkis \& Tryfonos, 2018). Existe la necesidad de enriquecer la base de conocimiento en cuanto a la impresión 3D de gran escala, y a la automatización de los procesos en la construcción impresa, encontrando soluciones tecnológicas de construcción aditiva y generando nuevas alternativas a un diseño optimizado (Al Jassmi, Al Najjar, \& Mourad, 2018). Se debe ahondar en la investigación que favorezca la comunicación directa del diseño paramétrico y la construcción impresa 3D con robots. 
Algunas Investigaciones experimentales en construcción impresa de viviendas permitieron con diseño paramétrico lograr la definición de capas y el establecimiento de recorridos precisos y eficientes. Con capas de $3 \mathrm{~cm}$ de altura y separación entre encuentros de $7 \mathrm{~cm}$ para favorecer la adherencia y no afectar a la resistencia estructural, en este caso, el modelo 3D de la vivienda se transformó a código G-code utilizando Grasshopper 3D. Previamente se usó Rhinoceros 3D para modelar la geometría de la vivienda (Sánchez Gómez et al., 2020). En el estudio se logró el aprovechamiento de la tecnología de impresión 3D con un diseño que permitía la deposición continua de hiladas de materiales con una longitud mínima de 2,7 m. De manera similar, otros autores utilizaron la programación paramétrica para el desarrollo de la impresión $3 \mathrm{D}$ en concreto de una estructura de forma compleja que fusiona un habitáculo cilíndrico con un techo cónico (Muthumanickam et al., 2020).

Otros autores desarrollaron un algoritmo paramétrico integrado para la planificación de trayectorias de herramientas y el control de extrusión, lo que permitió mediante un brazo robótico, la impresión 3D de piezas huecas de muros con doble curvatura con ángulos de inclinación en sus paredes de hasta de $26,13^{\circ}$ con respecto a la vertical, con y sin relleno celular interior optimizado con distintos materiales de impresión (Odysseas Kontovourkis \& Tryfonos, 2020). Alternativamente a la implementación de BIM y su vinculación con la construcción robotizada, un estudio presentó un diseño y programación paramétrica con Grasshopper para evaluar distintas configuraciones para paneles prefabricados de concreto con doble curvatura altamente complejos con generación de codificación GCode (Lim et al., 2016).

Considerando lo anterior, y dado que la construcción robotizada progresivamente se abre paso en la actualidad, su impacto con el tiempo será mayor conduciendo a las empresas de construcción a adaptarse a la robótica (Bakir \& Balchi, 2018). En ambientes donde coexistan humanos y robots serán necesarias secuencias de información para garantizar la seguridad y la productividad (Teizer et al., 2018).

La construcción impresa aún se encuentra en un estado experimental que requiere de masa crítica que posibilite su desarrollo y adopción. Con esta investigación se busca contribuir al respecto. Para ello, las tecnologías BIM son determinantes dada su aceptación actual y sus atributos en exploración y proyección formal para el diseño paramétrico, así como en programación paramétrica del modelo buscando comunicación directa con el robot. E conocimiento actual de las funciones que permiten los software BIM ha entregado nuevas aproximaciones para el desarrollo de procesos de fabricación automatizados en construcción. Gracias al diseño digital, los procesos de diseño, planificación y construcción han mejorado significativamente. Sin embargo, la aplicación de BIM para la impresión 3D aún está por crecer (Teizer et al., 2018).

Este artículo muestra un proceso de diseño y modelamiento paramétrico de muros curvos en BIM para impresión 3D en concreto, cuyo propósito es reducir las cantidades de materiales y tiempos de ejecución en la construcción, para posteriormente realizar la programación de su construcción impresa robotizada. Con ello se busca generar evidencia de la factibilidad del proceso para salvar la brecha existente actualmente entre los modelos, su información y su fabricación, de igual forma contribuir en procesos de diseño que aborden geometrías complejas..

\section{TRABAJOS RELACIONADOS}

La investigación aquí presentada forma parte de un proyecto interdisciplinario de desarrollo de construcción impresa usando brazos robóticos. Este estudio está integrado, entre otros, por los siguientes trabajos relacionados:

- Exploración formal con diseño paramétrico de geometrías complejas.

- Diseño integrado para modelamiento y programación para construcción impresa con brazo robótico.

- Calculo estructural de elementos o componentes impresos de concreto.

- Diseño de mezclas de concreto para construcción impresa.

- Programación y comunicación para automatización de brazo robótico.

- Optimización de diseños geométricos para construcción impresa.

La etapa de la validación estructural de muros curvos en concreto fue desarrollada a través de una tesis de pregrado de Ingeniería Civil de la Universidad del Bío Bío (Hidalgo-Quezada \& Valdebenito-Núñez, 2020). Particularmente se desarrolló el cálculo estructural de un muro curvo variando su curvatura y agudeza, según la resistencia al volcamiento en función de diferentes espesores de muro, además, mediante un estudio de optimización estructural se establecieron las curvaturas e inclinaciones posibles para los muros en función de su espesor, para esto se divide el muro patrón en porciones curvas al que se le realiza el análisis estructural usando el método numérico.

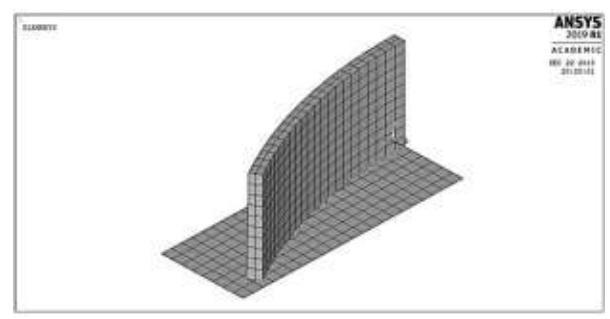

Figura 3: Modelo estructural del muro curvo. (Fuente: (HidalgoQuezada \& Valdebenito-Núñez, 2020).

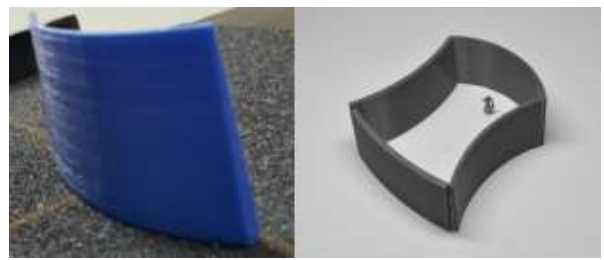

Figura 4: Muestras impresas a escala de muros curvos inclinados, verificados con analisis estructural. (Fuente: (HidalgoQuezada \& Valdebenito-Núñez, 2020)). 
Esto permite tomar precaución para el proceso de impresión con el brazo robótico, garantizando que no se produzca volcamiento del elemento. En la figura 3 podemos ver el modelo estructural desarrollado para la investigacion, en la figura 4 una muestras iniciales de impresión 3D en plastico de muros curvos.

De igual forma la etapa de programación para la comunicación entre la modelación BIM y el brazo robótico fue desarrollada con una tesis de pregrado de Ingeniería Civil de la Universidad del Bío Bío, en las que el desarrollo de muros impresos se logró, mediante programación paramétrica permitiendo generar trayectorias de impresión para brazos robóticos a partir de los elementos constructivos (Pérez-Ortiz \& Vásquez-Zúñiga, 2020).

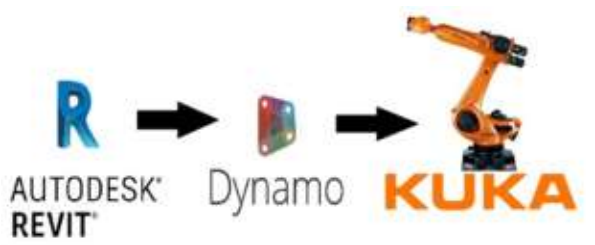

Figura 5: Esquema de vinculación del modelo BIM con el brazo robotico (Fuente: (Pérez-Ortiz \& Vásquez-Zúñiga, 2020)).

Conjuntamente con el presente trabajo dentro del proyecto mencionado, se realizó la programación paramétrica para generar un conjunto masivo y significativo de alternativas de diseño geométrico para resolver un recinto particular para pabellón de vehículos, con la combinación de paredes curvas impresas en 3D. Dado que la curvatura horizontal de los muros genera una mayor resistencia a las fuerzas de volcamiento, es posible reducir considerablemente su espesor, y como consecuencia el consumo de material.

Con todo esto se llevó a cabo una evaluación en función de los aspectos económicos que permitió la selección sobre 1600 alternativas que combinan muros curvos, alcanzando la reducción de su espesor sin afectar su resistencia a fuerzas de volcamiento.

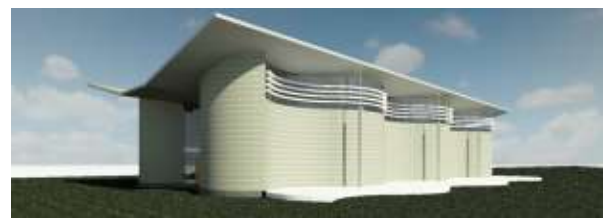

Figura 6: Prototipo de edificación con muros curvos verticales generado con el proceso descrito para esta fase. (Fuente: (Martínez-Rocamora, García-Alvarado, Casanova-Medina, González-Böhme, \& Auat-Cheein, 2020a)).

En dicho estudio se lograron destacadas reducciones de tiempos de construcción y de consumo de material (61\%) lo que permitió un ahorro de costos del 53\% (MartínezRocamora, García-Alvarado, Casanova-Medina, González-Böhme, \& Auat-Cheein, 2020b). La solución optimizada del recinto con el desarrollo arquitectónico posterior puede verse en la figura 6.
El presente artículo se centra en el desarrollo de las familias paramétricas BIM de los muros en relación a los trabajos aquí mencionados, con el fin de conducir a completar el proceso de programación paramétrica de la construcción impresa con el brazo robotizado.

\section{MATERIALES Y MÉTODOS}

El desarrollo de la investigación se llevó a cabo con las siguientes fases:

- Fase conceptual de exploración preliminar de diseño, definiendo parámetros formales en Revit ${ }^{\mathrm{TM}}$ : el módulo básico del modelo geométrico del muro lo conforman dos arcos de tres puntos en su proyección en planta (plano $X Y$ ), opuestos y unidos en uno de sus extremos, que corresponden al eje del elemento. Estos son extruidos para generar el espesor del muro. Para el plano base, definiendo el elemento 1 (arco 1), los puntos A, B y C, y el elemento 2 (arco 2), los puntos $\mathrm{C}$, D y E, siendo $\mathrm{C}$ común a los dos elementos. Las variaciones de la cuerda para el arco 1 (distancia $A C$ ), obedecen al desplazamiento en $X$ izquierda (-X) del punto $C$, en las posiciones 1,2 y 3 , así también al movimiento del punto $B$ en la misma dirección.

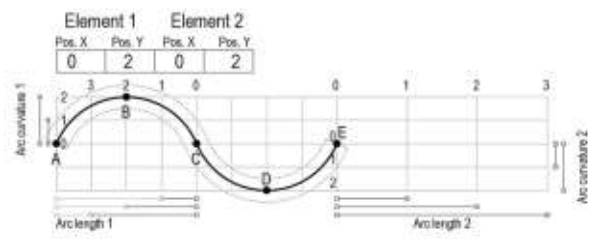

Figura 7: Variaciones de posición de los puntos sobre el eje de los elementos en el plano base $X Y$.

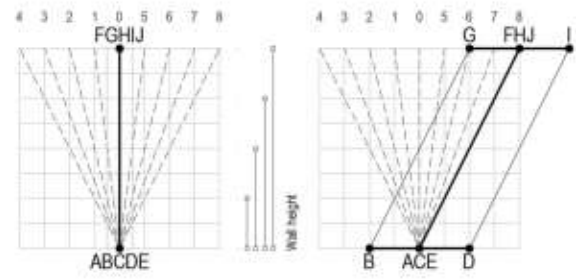

Figura 8: Inclinación lograda con la variación de la posición de los puntos de los arcos sobre el eje de los elementos para dos tipos de muros en el plano YZ (Autores).

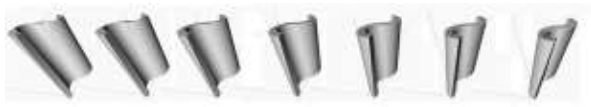

Figura 9: Muro curvo con varias alternativas de inclinación (Autores).

Para la variación de la cuerda para el arco 2, el punto que se desplaza es el $E$ en dirección $X$ derecha $(+X)$, en las posiciones 1,2 y 3 , así también al movimiento del punto $D$ en la misma dirección (Figura 7). 
La curvatura del arco 1 varia con el desplazamiento del punto $B$ en el eje $Y$ en dirección positiva $(+Y)$, y para el arco 2 con el desplazamiento del punto $D$ en dirección $Y$ negativa $(-Y)$, en sus respectivas posiciones 1 y 2 . La inclinación del muro se logra con el desplazamiento de la replicación de estos puntos en un plano superior, denominándose $\mathrm{F}, \mathrm{G}, \mathrm{H}, \mathrm{I}$ y J. Los parámetros del muro para su variación con inclinación pueden observarse en la figura 8. La inclinación se produce por el desplazamiento horizontal del plano que contiene a los elementos 1 y 2 a una altura $h$, en dirección perpendicular al eje AE del plano base.

Para la presente investigación la exploración formal del proceso contempla conservar la misma forma de los elementos 1 y 2 , con puntos ABCDE, en el plano base y puntos FGHIJ en el plano a una altura h, generando una superficie al conectar los arcos en distintas alturas, y un volumen al proporcionarle un espesor a dicha superficie. La altura del muro corresponde a la diferencia en cota de los dos planos mencionados. La sección transversal del muro en el plano vertical es constante y rectangular, siendo posible variar su ancho para explorar múltiples alternativas de espesor. Una configuración de muro y espesor con distintas opciones de inclinación puede apreciarse en la figura 9 .

Para un control de combinaciones se creó una codificación que permite identificar cada alternativa según los parámetros definidos y sus variaciones restringidas. Este control mediante códigos resulta esencial dado que, para una altura y espesor fijos, y con posición vertical del muro, es posible explorar un total de 144 alternativas de configuración, y con la opción de inclinación un total de 1152 posibilidades de muro patrón base.

- Fase de modelamiento paramétrico con Dynamo ${ }^{\mathrm{TM}}$ y Revit ${ }^{\mathrm{TM}}$ : se desarrolla la familia paramétrica del muro curvo e inclinado en BIM, partiendo de los parámetros anteriores usando Dynamo ${ }^{\mathrm{TM}}$.

El proceso inicia con la programación en Dynamo $^{\mathrm{TM}}$ de los parámetros y variaciones anteriormente descritos, definiendo la programación visual de los parámetros de la geometría del muro curvo e inclinado, generando el script correspondiente.

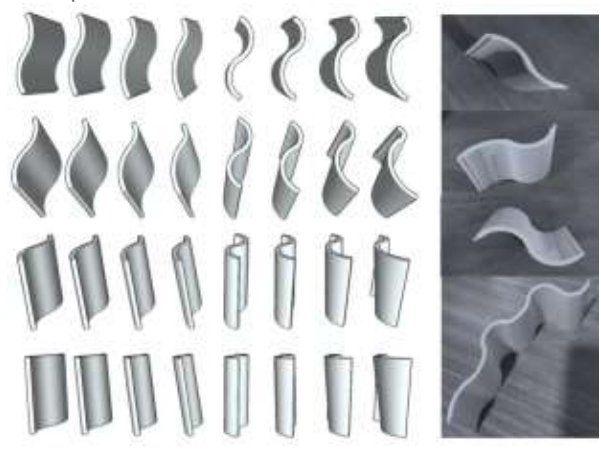

Figura 10: Distintas opciones de combinación de parámetros para muros curvos inclinados y algunas muestras impresas.
Con la codificación definida anteriormente, que obedece a la variación de los parámetros de la configuración geométrica, se identifica cada alternativa de muro patrón, lo cual es clave para la creación de la familia paramétrica de los muros curvos en BIM. Una exploración en diseño con aplicación combinada de distintas alternativas de muro patrón se plantea en la figura 10 , se muestran algunas opciones de combinación de muros patrón con la variación de los arcos de su eje. En la figura 11 se muestra una aplicación de diseño paramétrico utilizando el proceso anteriormente planteado para los muros curvos generando alternativas geométricas para un recinto particular.

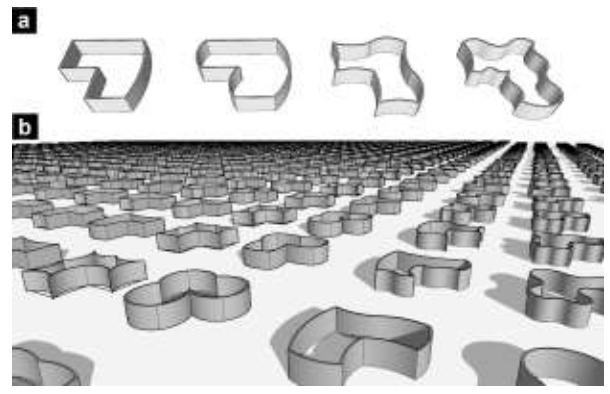

Figura 11: Generación automática de 7800 alternativas usando muros curvos para soluciones geométricas de muros en un recinto con planta en $L$ de $12 \times 12 \mathrm{~m}$. En la figura 11 a se muestra las configuraciones básicas del proceso y en la figura $11 \mathrm{~b}$ una vista general del conjunto de soluciones con muros curvos.

- Fase de integración de la validación estructural y programación paramétrica de la trayectoria del brazo robótico, y ejecución con construcción impresa, basada en el análisis de casos representativos y selección de casos para impresión:

Con el proceso de diseño y programación paramétrica de la fase anterior e integrando lo desarrollado por (PérezOrtiz \& Vásquez-Zúñiga, 2020), se define el elemento curvo para construcción impresa $3 \mathrm{D}$, el proceso transforma el volumen del elemento y lo descompone en sus superficies envolventes, determinando un eje de posición y altura de corte para generar un trazado helicoidal. La secuencia de puntos de la trayectoria debe ser regulada de acuerdo a la complejidad de la geometría y la velocidad de impresión para asegurar una ejecución expedita y precisa. Debe regularse además, la altura de separación o desfases verticales y ángulos de esquinas, calibrando todos estos valores en pruebas preliminares de impresión, de acuerdo a la mezcla utilizada y condiciones de humedad vigentes. También de acuerdo al alcance geométrico del brazo robótico o rieles de sujeción de la boquilla debe segmentarse el volumen. Así mismo, se deben definir los puntos de origen y de término de las trayectorias, según las etapas de ejecución y las capacidades de las bombas de alimentación.

En las pruebas de ejecución realizadas (ver Fig. 13) se advierte también que la velocidad incide en la rugosidad del cordón debido a la fluidez de la mezcla. Con ello se demuestra la factibilidad de vincular la modelación BIM con la impresión robotizada, pero también la necesidad de regular y calibrar los parámetros específicos para su ejecución. 
Finalmente se realizan pruebas preliminares de impresión del modelo seleccionado para verificar la validez del procedimiento y ajustar los procesos de ser necesario.

\section{RESULTADOS Y DISCUSIÓN}

El desarrollo y análisis de soluciones de diseño logradas con el modelado y programación BIM de muros de hormigón curvos para construcción impresa anterior puede verse en la figura 12 .

Las muestras iniciales impresas en plástico a escalas reducidas (figura 10), mostraban los posibles inconvenientes que presentarían los muros al ser construidos con el brazo robótico a escala humana: desfases de capas, falta de adherencia con la base, falta de adherencia entre capas, barrido de la boquilla sobre el material impreso que podría deformarlo o volcarlo, o falta de continuidad de la línea de impresión. Todos estos aspectos fueron analizados y evaluados para su corrección.

La verificación estructural previa sirvió para determinar la máxima inclinación posible para una curvatura y espesor de muro, lo que permite llevar a cabo el proceso de impresión 3D del muro con una menor incertidumbre ante la posibilidad de volcamiento. De igual forma el diseño previo de la mezcla de concreto permite controlar la fluidez, plasticidad y fraguado del concreto para evitar asentamientos y deformaciones excesivas que interrumpan o perturben el proceso de impresión y el producto final.

Fue necesario programar el recorrido del brazo robótico con las precauciones anteriores, planificando una trayectoria óptima que redujera los tiempos de impresión. Esta trayectoria debe ser homogénea y continua, alimentada constantemente por la bomba impulsora de concreto. La muestra impresa lograda evidencia la efectividad del diseño y programación paramétrica para este tipo de muro. El tiempo promedio de impresión para

a

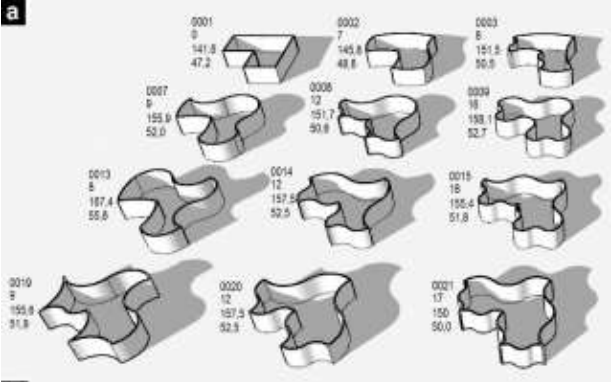

una pieza a escala humana es de 10,5 minutos, un $20 \%$ del tiempo que requeriría su construcción con técnicas y tecnologías tradicionales. El proceso puede verse en la figura 13 .

La utilidad del diseño paramétrico es aprovechada en esta investigación como herramienta de exploración formal para soluciones arquitectónicas, la automatización para la generación masiva de alternativas permite disponer de un amplio repertorio de opciones en la configuración espacial, lo que resulta conveniente en la reducción de tiempos de diseño, al igual que de construcción, al apoyarse en procesos adicionales de optimización ya sea, estructural, de costos, energética o ambiental, la herramienta de diseño paramétrico se convierte en un apoyo en tomas de decisiones para proyectos arquitectónicos. Por otro lado, la exploración formal realizada y la solución del proceso de comunicación con el robot contribuyen en la aplicabilidad de soluciones geométricas no convencionales y en la factibilidad de su construcción impresa.

Estos resultados y sus comparaciones con otras investigaciones muestran la factibilidad del proceso considerado que permiten facilitar la comunicación con el brazo robotizado, optimizando el tiempo de interacción diseño-planificación-construcción.

\section{CONCLUSIONES}

Este trabajo presenta una metodología para la programación paramétrica usando BIM, que permite aprovechar las ventajas que ofrece la tecnología de impresión 3D, particularmente en la exploración de diseños de muros curvos que permiten combinaciones múltiples en arquitectura.

La programación paramétrica realizada permite vislumbrar un amplio rango de opciones de configuración geométrica para cerramiento en una edificación, aunque la transformación geométrica de cada muro se limita a modificaciones con rangos preestablecidos que obedecen

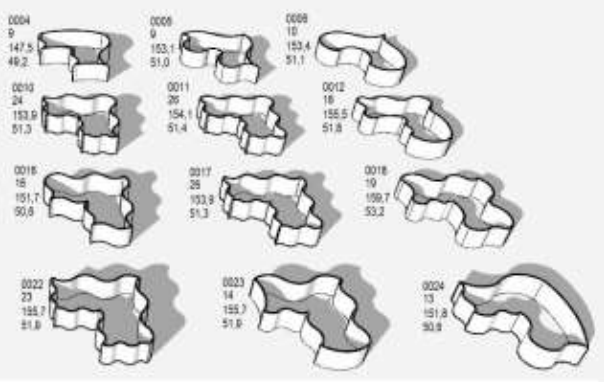

b

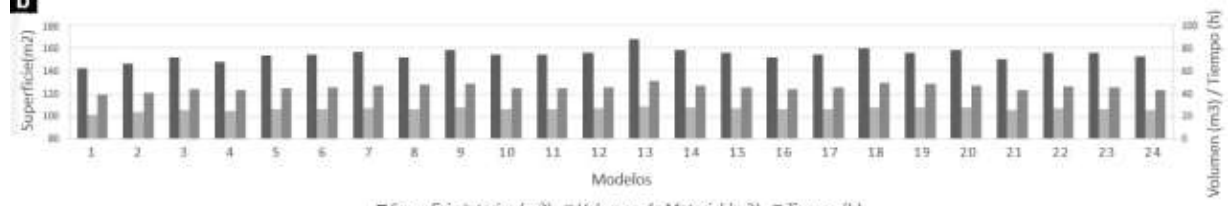

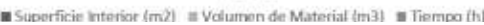

Figura 12: Aplicabilidad del diseño paramétrico para construccion impresa 3D mediante Dynamo en un recinto en $L$ de dimensiones $12 \times 12$ m en sus lados mayores. La figura 13a es el repertorio seleccionado de soluciones para el recinto, y la figura $13 \mathrm{~b}$ muestra la superficie interior, el volumen de material de cada alternativa y los tiempo de impresión. 
a la codificación definida del elemento para facilitar su programación. Alternativamente con cambios mínimos de configuración sobre las variaciones geométricas por fuera de los rangos definidos es posible generar un abanico muy amplio de combinaciones.

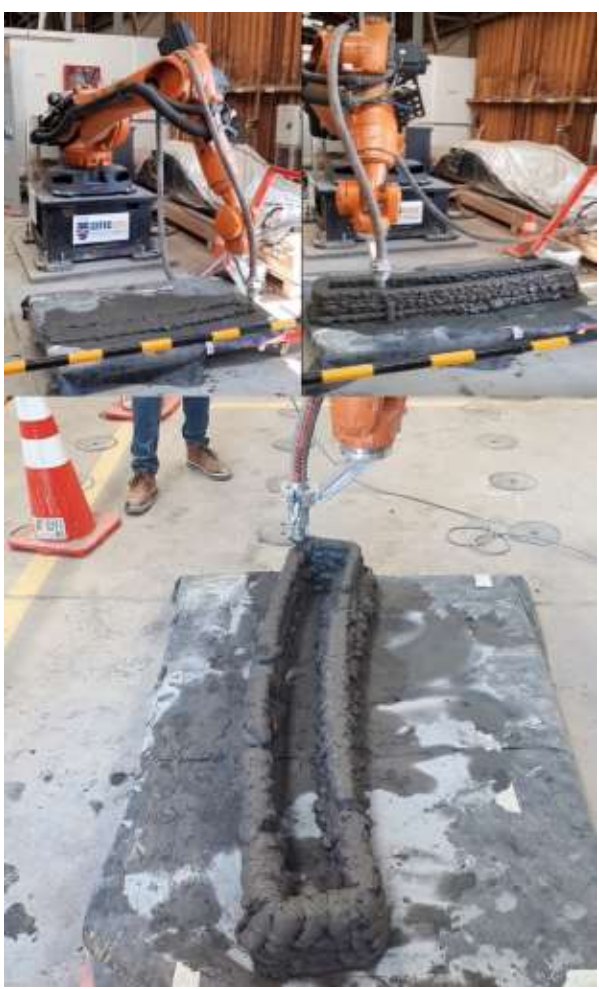

Figura 13: Construcción Impresa 3D de muro curvo con Robot KukaR120 y Bomba de Hormigonado (Autores).

Las ventajas más relevantes que ofrece la construcción impresa comparativamente con la construcción tradicional son los tiempos reducidos de construcción, mínimos desperdicios de material, el control en los procesos de construcción, y la seguridad de la ejecución por no poner en riesgo a obreros. Además de lo anterior, con la presente investigación se corrobora la versatilidad de la tecnología, con la que es posible desarrollar cualquier forma y construirla, todo esto es posible gracias a la integración que se puede lograr entre BIM, fabricación digital y elementos robotizados, con el diseño y programación paramétrico que contribuye a fortalecer su interacción y potenciar su utilidad a partir de la integración de proceso.

Los resultados logrados con la impresión de las muestras seleccionadas e impresas muestran la factibilidad de la técnica estudiada y de la metodología propuesta, contribuyendo a la compilación de evidencias de las ventajas de la tecnología de construcción impresa 3D que promuevan su desarrollo progresivo, pero principalmente su adopción.
El proceso desarrollado en esta investigación puede ser aplicable en soluciones con mayor complejidad, realizando variaciones en la sección transversal de los muros como inclinaciones, curvaturas verticales, irregularidades formales en la sección y las combinaciones de estas alternativas. Este trabajo hace un aporte a la exploración de procesos de comunicación entre diseño digital y el brazo robotizado para construcción impresa 3D con hormigón.

Dentro de las futuras investigaciones desarrolladas a partir del proyecto se encuentran:

Diseño y programación paramétrica para la construcción de elementos de doble curvatura, incluyendo junto con estas, las alternativas de variación formal con inclinaciones, curvaturas verticales y sus combinaciones. Por otra parte, estudiar la contructibilidad de elementos curvos en inclinados mediante construcción impresa. Llevar a cabo una exploración en el diseño de mezclas para construcción impresa aplicables a la construcción de formas complejas. Aportar en aspectos estructurales con la exploración el desarrollo de sistemas estructurales acordes a la tecnología de construcción impresa 3D. A partir de todo lo anterior, lograr una aplicación del diseño y programación paramétrica para una solución habitacional completa realizada mediante construcción impresa 3D.

\section{AGRADECIMIENTOS}

Los autores agradecen a la Agencia Nacional de Investigación y Desarrollo de Chile (ANID) por apoyo del proyecto FONDECYT 1181015 y al Centro Interdisciplinario de Productividad y Construcción Sustentable (CIPYCS) de la U. del Bío-Bío.

\section{REFERENCIAS}

Al Jassmi, H., Al Najjar, F., \& Mourad, A. H. I. (2018). Large-scale 3D Printing: The way forward. IOP Conference Series: Materials Science and Engineering, 324(1). https://doi.org/10.1088/1757-899X/324/1/012088

Bakir, A., \& Balchi, I. (2018). Development and implementation of robotics in construction. A case study of a contractor firm. Chalmers University of Techonology.

Berman, B. (2012). 3-D printing: The new industrial revolution. Business Horizons, 55(2), https://doi.org/10.1016/j.bushor.2011.11.003

Bos, F., Wolfs, R., Ahmed, Z., \& Salet, T. (2016). Additive manufacturing of concrete in construction: potentials and challenges of 3D concrete printing. Virtual and Physical Prototyping, 11(3), 209-225. https://doi.org/10.1080/17452759.2016.1209867

Buswell, R. A., Soar, R. C., Gibb, A. G. F., \& Thorpe, A. (2007). Freeform construction: Mega-scale rapid manufacturing for construction. Automation in Construction, 16(2), 224-231. https://doi.org/10.1016/j.autcon.2006.05.002

Cho, S., Kruger, J., Zeranka, S., \& Zijl, G. Van. (2019). 3D Printable concrete technology.

Coulter, F. B., Coulter, B. S., Papastavrou, E., \& lanakiev, A (2018). Production techniques for 3D Printed inflatable elastomer structures: Part II-Four-axis direct ink writing on irregular double-curved and inflatable surfaces. 3D Printing and Additive Manufacturing, 5(1), 17-27. https://doi.org/10.1089/3dp.2017.0069

Craveiro, F., Nazarian, S., Bartolo, H., Bartolo, P. J., \& Pinto Duarte, J. (2020). An automated system for 3D printing functionally graded concrete-based materials. Additive 
Manufacturing,

https://doi.org/10.1016/j.addma.2020.101146

Cui, Q., \& Yue, F. (2020). Parametric design of personalized 3D Printed sneakers. In Proceedings of the 2019 Digital Futures (pp. 82-92). Springer Singapore. https://doi.org/10.1007/978-981-13-8153-9

García-Alvarado, R. (2018). Initial developments and projections of $3 \mathrm{D}$ Construction printing. In ARCC Conference Repository.

Gosselin, C., Duballet, R., Roux, P., Gaudillière, N., Dirrenberger, J., \& Morel, P. (2016). Large-scale 3D printing of ultra-high performance concrete - a new processing route for architects and builders. Materials and Design, 100, 102109. https://doi.org/10.1016/j.matdes.2016.03.097

Hager, I., Golonka, A., \& Putanowicz, R. (2016). 3D Printing of buildings and building components as the future of sustainable construction? Procedia Engineering, 151, 292299. https://doi.org/10.1016/j.proeng.2016.07.357

Hidalgo-Quezada, N. E., \& Valdebenito-Núñez, M. I. (2020). Análisis de volcamiento de muros curvos con diferentes geometrias para construcción aditiva. Memoria de título en Ingeniería Civil. Universidad del Bío Bío.

Izard, J.-B., Dubor, A., Hervé, P.-E., Cabay, E., Culla, D., Rodriguez, M., \& Barrado, M. (2017). Large-scale 3D printing with cable-driven parallel robots. Construction Robotics, 1(1-4), 69-76. https://doi.org/10.1007/s41693017-0008-0

Kontovourkis, O., \& Tryfonos, G. (2018). Integrating parametric design with robotic additive manufacturing for 3D clay printing: An experimental study. ISARC 2018 - 35th International Symposium on Automation and Robotics in Construction and International AEC/FM Hackathon: The Future of Building Things, (Isarc). https://doi.org/10.22260/isarc2018/0128

Kontovourkis, Odysseas, \& Tryfonos, G. (2020). Robotic 3D clay printing of prefabricated non-conventional wall components based on a parametric-integrated design. Automation in Construction, 110(November 2019), 103005. https://doi.org/10.1016/j.autcon.2019.103005

Lim, S., Buswell, R. A., Valentine, P. J., Piker, D., Austin, S. A., \& De Kestelier, X. (2016). Modelling curved-layered printing paths for fabricating large-scale construction components. Additive Manufacturing, 12, 216-230. https://doi.org/10.1016/j.addma.2016.06.004

Martínez-Rocamora, A., García-Alvarado, R., Casanova-Medina, E. González-Böhme, L. F., \& Auat-Cheein, F. (2020a). Parametric programming of $3 D$ printed curved walls for cost-efficient building design. Journal of Construction Engineering and Management, 146(5), 1-13. https://doi.org/10.1061/(ASCE)CO.1943-7862.0001811

Martínez-Rocamora, A., García-Alvarado, R., Casanova-Medina, E., González-Böhme, L. F., \& Auat-Cheein, F. (2020b). Parametric programming of $3 \mathrm{D}$ Printed curved walls for cost-efficient building design. Journal of Construction Engineering and Management, 146(5), 1-13. https://doi.org/10.1061/(ASCE)CO.1943-7862.0001811

Mechtcherine, V., Nerella, V. N., Will, F., Näther, M., Otto, J., \& Krause, M. (2019). Large-scale digital concrete construction - CONPrint3D concept for on-site, monolithic 3D-printing. Automation in Construction, 107(August), 102933. https://doi.org/10.1016/j.autcon.2019.102933

Monedero, J. (2000). Parametric design: A review and some experiences. Automation in Construction, 9(4), 369-377. https://doi.org/10.1016/S0926-5805(99)00020-5

Muthumanickam, N. K., Park, K., Duarte, J. P., Nazarian, S., Memari, A., \& Bilén, S. G. (2020). BIM for parametric problem formulation, optioneering, and 4D simulation of 3Dprinted Martian habitat: A case study of NASA's 3D Printed Habitat Challenge. In 5th Residential Building Design \&
Construction (RBDC). State College, PA.

Nair, S. A. O., Panda, S., Santhanam, M., Sant, G., \& Neithalath, N. (2020). A critical examination of the influence of materia characteristics and extruder geometry on 3D printing of cementitious binders. Cement and Concrete Composites, 112 ,

103671 https://doi.org/10.1016/j.cemconcomp.2020.103671

Pérez-Ortiz, J. I., \& Vásquez-Zúñiga, Á. G. (2020). Protocolos de comunicación entre modelos BIM y hormigones impresos en $3 D$. Memoria de título en Ingeniería Civil. Universidad del Bío Bío.

Perkins, I., \& Skitmore, M. (2015). Three-dimensional printing in the construction industry: A review. International Journal of Construction Management 15(1), 1-9. https://doi.org/10.1080/15623599.2015.1012136

Salminen, S., \& Tallberg, T. (1996). Human errors in fatal and serious occupational accidents in Finland. Ergonomics, 39(7), https://doi.org/10.1080/00140139608964518

980-988.

Sánchez Gómez, S., Alapont Ramón, J. L., Bono Cremades, J., \& Catalán Tamarit, F. J. (2020). Printed building in Morocco 3D Printed concrete prototype for the Solar Decathlon Africa 2019. In $9^{\circ}$ Congreso Internacional de Arquitectura Blanca - CIAB (pp. 298-305). Valencia: Universidad Politécnica de Valéncia.

Sorock, G. S., O'Hagen Smith, E., \& Goldoff, M. (1993). Fatal occupational injuries in the New Jersey construction industry, 1983 to 1989. Journal of Medicine, 35, 916-921.

Suzuki, R., Yatani, K., Gross, M. D., \& Yeh, T. (2018). Tabby : Explorable design for 3D Printing textures, 2-5.

Tay, Y. W. D., Panda, B., Paul, S. C., Noor Mohamed, N. A., Tan, M. J., \& Leong, K. F. (2017). 3D printing trends in building and construction industry: a review. Virtual and Physical Prototyping, 12(3), 261-276. https://doi.org/10.1080/17452759.2017.1326724

Teizer, J., Blickle, A., King, T., Leitzbach, O., \& Guenther, D. (2016). Large scale 3D printing of complex geometric shapes in construction. ISARC 2016 - 33rd International Symposium on Automation and Robotics in Construction, i(Isarc), 948-956. https://doi.org/10.22260/isarc2016/0114

Teizer, J., Blickle, A., King, T., Leitzbach, O., Guenther, D. Mattern, H., \& König, M. (2018). BIM for 3D printing in construction. Building Information Modeling: Technology Foundations and Industry Practice, 421-446. https://doi.org/10.1007/978-3-319-92862-3_26

Uppala, S. sandeep, \& Tadikamalla, M. (2017). Review paper a review on 3D Printing of concrete - the future of sustainable construction. I-Manager's Journal on Civil Engineering, 7(3), 2017.

Wolfs, R. J. M., \& Suiker, A. S. J. (2019). Structural failure during extrusion-based 3D printing processes. International Journal of Advanced Manufacturing Technology, 104(1-4) 565-584. https://doi.org/10.1007/s00170-019-03844-6

Yin, H., Qu, M., Zhang, H., \& Lim, Y. C. (2018). 3D Printing and buildings: A technology review and future outlook. Technology Architecture and Design, 2(1), 94-111. https://doi.org/10.1080/24751448.2018.1420968

Zareiyan, B., \& Khoshnevis, B. (2017). Interlayer adhesion and strength of structures in Contour Crafting - Effects of aggregate size, extrusion rate, and layer thickness. Automation in Construction, 81(June), 112-121. https://doi.org/10.1016/j.autcon.2017.06.013 